\title{
Application of the Flipped Classroom in Medical Physics Teaching Guangxiao $\mathrm{He}^{1,}$ a $\mathrm{Li} \mathrm{Li}^{2}$, Pengcheng Zhang ${ }^{1}$, Benchao $\mathrm{Zhu}^{1}$, Jia Guo Kang ${ }^{1}$ and
}

${ }^{1}$ Department of Mathematics and Physics, Hubei University of Medicine, Shiyan 442000, China; ${ }^{2}$ Checheng High School, Shiyan 442000, China.

ahgx@hbmu.edu.cn, bwbkang@hbmu.edu.cn

*The corresponding author

\begin{abstract}
To explore the preliminary effects of the flipped classroom in medical physics teaching. The students were from two undergraduate classes of the Grade 2015 in Hubei University of Medicine as research object. The one class is considered as an experimental group, another class for the control group. The teaching effect of the two groups was compared and analyzed. The overall theoretical level and practical ability of the experimental group were significantly higher than the control group. It is practicable to apply flip classroom to medical physics teaching. The flip classroom meets the students' personalized learning, improve the comprehensive quality of students and improve the teaching effect of teachers. Flip classroom is conducive to promoting the basic teaching reform and transformation development of local medical college under new situation.
\end{abstract}

Keywords: Flipped classroom; Medical physics; Innovation; Teaching model

\section{翻转课堂在《医学物理学》教学中的应用}

\author{
何光晓 ${ }^{1}$, 李丽 ${ }^{2}$, 张鹏程 ${ }^{1}$, 朱本超 ${ }^{1}$, 郭佳 ${ }^{1}$, 康文斌 ${ }^{1 *}$
}

(1. 湖北医药学院、数理教研室, 湖北十堰 $442000 ; 2$. 车城高级中学, 湖北十堰 442000)

摘要: 探究翻转课堂在医学物理学教学中的初步教学效果。选取湖北医药学院 2015 级临床医学专业两个本科班作为研 究对象。一个本科班作为实验组, 另一个本科班为对照组。对两组教学效果进行对比分析。实验组的整体理论水平和动手能 力明显高于对照组。将翻转课堂应用于医学物理学教学中切实可行。翻转课堂满足学生的个性化学习, 提升学生综合素养, 提高教师教学效果。翻转课堂有利于推动新形势下地方医学院校基础教学改革和转型发展。

关键词: 翻转课堂; 医学物理学; 创新; 教学模式

中图分类号：TM 344.1 文献标志码：A

引言

现代社会医学模式对医学服务提出新的挑战, 这要求未来的医学人才不仅要具有创新的医学实践能 力, 还要具备深厚的物理知识。医学物理学是把物理学原理和方法应用于医学研究与临床实践中的一门 交叉学科。医学物理学为培养全方位高水平的应用型医学人才提供了丰富的物理学知识 ${ }^{[1]}$ 。

随着时代发展与科技进步, 高等教育要求教学理念和方式不断创新。传统的单向知识传输教学、学生 被动学习的教学模式多被病诟。如何改进医学物理课程教学, 是医学院校教师们不断努力思考和亟待解决 的问题。近年来, 有研究者介绍了国内外《医学物理学》教学现状, 同时还为学科发展提出了宝贵的意见 和建议 ${ }^{[2-3]}$ 。也有研究者探索出了许多新的教学模式 (例如: 案例式、探究式、讨论式、窗口式、目标型、 任务型、开放型、双向互动型、非线性、PBL、STS、双语等) 在物理教学中的实践与应用 ${ }^{[4-5]}$ 。还有研究者 将一些现代技术 (例如: Internet、Flash、Matlab、AutoCAD、Solidworks、Origin 等) 融入到物理教学 
中。这些方法和技术对提高物理教学效果起到一定的促进作用。随着计算机网络的广泛普及, 教学形式已 不再局限于课堂教学。在 “互联网 + ” 的浪潮下, 翻转课堂在高等教育教学中的应用已多手可热 ${ }^{[6]}$ 。我国北 京大学、清华大学、复旦大学、上海交通大学等许多知名大学都已开始实施基于翻转课堂的教育教学创新。 翻转课堂让学生充满了兴趣并愿意去亲身体验。新的教学模式有利于激发学生学习的兴趣, 调动学生学习 的积极性, 提高学生自主探究能力, 培养医学生的理科思维。随着翻转课堂的推广, 也将促进教师教学、 管理、设计能力的发展, 教学理念、方法、手段的转变, 专业、非专业知识的更新, 实现教师为主导, 学 生为主体的课堂教学格局, 推动课程的教育创新升级 ${ }^{[7]}$ 。

\section{1 传统的医学物理教学模式分析}

传统的物理教学模式以教师、书本、课堂为中心，教学方法单一。高校招生数量急剧增长，学生基础 参差不齐, 教学资源出现相对短缺。传统的教学方法使得物理课堂变得比较枯燥乏味且需要大量的记忆和 思考, 大多数同学对此不感兴趣, 个别学生课堂上睡觉或玩移动终端, 学生学习热情低、参与度低、学习 效率低, 应付考试, 凑学分等问题尤为突出。究其原因有以下几点: 第一, 部分医学生认为自己非物理专 业, 对物理学的作用不够重视, 缺乏学习动力; 第二, 学生接受以往灌输式学习, 不愿意变被动学习为主 动性学习; 第三, 部分学生未能掌握正确的学习方法; 第四, 医学物理学课程学分相对较高, 迫于学分的 压力而选择重修本课程。

\section{2 翻转课堂在医学物理中的教学实践}

为了更好的解决以上题, 改善传统教学的若迫局面, 故采用翻转课堂教学模式对医学物理学课程进行 改革和探究。基于校课程中心网络平台和学科网站, 探索翻转课堂应用于《医学物理学》教学。对《医学 物理学》教材中的教学内容进行整合, 以专题为模块开展翻转课堂。选择 2015 级临床专业两个班级进行 教学 “试点”, 以甲班作为试验样本, 乙班作为对照样本, 比较两种教学模式的教学效果。

\section{1 教学内容整合}

认真研究医学生的培养目标与课程的教学目标, 并结合以往教学实践经验, 对医学物理学中与医学关 系密切的教学内容进行整合, 将表 1 中的内容作为翻转课堂的试点。表 1 中选择的内容既高度 “浓缩” 了 课程的重点, 提高了课程的教学质量 ${ }^{[8-9]}$, 又为学生后续的专业课程的学习奠定了理论基础。翻转课堂一般 在一个学期的中后期开展, 教学模式的转换, 以学生为中心, 最大限度集中学生注意力, 提升学生课堂自 信心，让学生获得 “新鲜感” 的同时汲取更多的医用物理学知识, 让学生能够学以致用。

表 1 医学物理学翻转课堂教学内容设计

\begin{tabular}{llll}
\hline 章节名称 & \multicolumn{1}{c}{ 讲授内容 } & \multicolumn{1}{c}{ 教学策略 } \\
\hline 力学基础 & 骨的力学性质探究 & 图片展示骨的受力 & 观看并试运用力学知识进行受力分析 \\
声 波 & 超声波的生物医学效应 & 三维动态超声视频 & 观看并思考超声波的原理、作用 \\
流体的运动 & 流体力学在血管壁生理中的应用 & 引入颈动脉狭窄案例 观看并思考其中的物理思想 \\
分子动理论 & 肺表面活性物质在呼吸过程中的作用 & 动画展示分子运动 & 观看并思考其中的物理过程 \\
热力学基础 & 在人体热力学中基础代谢率对疾病诊断的意义 & 以问题引导学习 & 思考物理原理在医学中的应用 \\
电场与电流 & RC 电路及其在神经传导中的应用 & 图片展示 RC 电路 & 观看并体会其中的物理方法 \\
磁场与电磁感应 & 心、脑、肺磁图在临床诊断中的应用 & 图片展示心、脑、肺 & 思考利用物理知 \\
& & 磁图 & 识为医学服务 \\
& & &
\end{tabular}


首先, 选择 2015 级临床医学专业中的两个本科班作为研究对象。其中开展翻转课堂教学的一个班为 实验组 (甲班), 实施传统教学的另一个班为对照组 (乙班)。甲班中的 48 名学生进行分组, 分 8 个小组, 每组 6 人。乙班学生人数为 51 人。甲班学生可根据表 1 中的内容, 选择自己感兴趣的模块进行自主学习, 但保证表 1 中的专题均被选择。学生可利用校内课程网站中心的资源以及网络资源进行线上学习，也可利 用学校图书资源进行线下学习。其次, 学生以小组为单位进行自主学习, 时间为两个星期, 学习完成后用 2 个学时来开展 $10 \sim 15$ 分钟的学习汇报, 交流学习成果。学生要跟所讲授内容自主制作 PPT 课件, 自我组 织课堂, 精心设计问题, 巧妙设计板书, 自行组织试讲。教师正式上课时, 从小组成员中任意指定一名同 学作为代表, 讲授所选专题。学生在讲授的过程中, 教师要做好记录。老师和学生的角色进行互换, 由学 生主宰课堂。讲授者与老师、同学进行交流互动, 从而拓展学生知识的深度和广度。学生在自主学习的过 程中教师要为学生获取与利用资源、处理信息与应用理论知识提供帮助。为了让学生更好进行个性化自主 学习, 教研室的 5 位医学物理学课程主讲教师已将部分基础理论的教学视频上传至学校课程网站中心, 课 程网站中心也有外校优秀开放教学资源的链接网址。教师转化为学生学习的指导者与助推者。最后, 每一 名小组代表讲授完成后，教师针对每一位学生所讲授的内容进行查缺补漏，锦上添花。

学生代表是随机选取的, 即要求小组的每一个成员认真地去学习、观察、分析、思考问题, 进行独立 探索, 独立构建自己的知识体系, 完成知识内化。这一举措可避免某些同学不劳而获、不假思索。他们在 探索的过程中可能会遇到不能独立解决的问题, 这就需要他们与小组的其他成员进行合作学习。通过对话、 商讨、争论进行思维的碰撞出智慧的 “火花”。在合作学习过程中有益于培养学生的沟通能力与团队精神。 在观点相佐时的问题解决有利于培养学生的包容能力与批判思维。在 PPT 课件的制作过程中可以培养学生 的动手能力与创新能力。

\section{3 教师点评与小结}

学生在进行学习汇报时, 邀请同行的高、中、初级职称的教师参与点评。学生讲解的过程中, 教师要 善于捕捉学生讲授精彩的地方, 同时也要详实地记录学生讲解过程中存在的问题和忽略的重点知识。教师 点评时先对学生展现的优点进行表扬, 以鼓励学生继续努力, 激发学生学习热情, 提高学生自信心和成就 感。然后对学生讲授过程中存在的问题予以纠正。对学生讲授中难点知识讲解不透彻的地方进行补充。对 学生讲授知识点的过渡不自然和衔接不紧密的地方提出改进意见和建议。鼓励学生在讲授时多与其他同学 进行互动交流。对学生讲课中重点不突出的地方进行强调。总之, 一切以提高学生能力和知识水平为目标, 促进学生的发展。

所有小组讲授完成之后, 教师应对本节课每一个小组所讲授内容总结出重点知识。帮助学生理清思路, 把握要点, 起到画龙点睛的作用。教师在后续教学中要通过随机抽查的方式检测学生对翻转课堂中知识的 掌握情况, 同时要求学生能够将翻转课堂中掌握的理论知识应用于实践, 还要对其他小组代表讲授中存在 的问题进行分析, 并能够提出解决问题的策略。即有利于培养学生分析问题, 解决问题的能力。

\section{3 翻转课堂教学实践的效果评价 ${ }^{[10]}$}

在 2015 级临床医学专业甲班的医学物理学教学中, 开展了翻转课堂教学模式的实践。在实践过程中 学生充满兴趣, 积极参与, 认真准备, 认真思考, 愿意亲身体验。翻转课堂结束后随机抽取甲班各个分组 中的 1 2 名学生, 对抽取的 12 名学生进行约谈。学生对医学物理学中开展翻转课堂的教学方法表示欢迎 
和支持。他们对于翻转课堂的教学持有以下的观点: 一、可以激发学生的学习热情; 二、能够活跃课堂氛 围; 三、提高学生参与度及课堂积极性; 四、提高学生上课的注意力; 五、实现学生研究性学习与个性化 自主学习; 六、提升学生对理论知识的理解; 七、提高已掌握知识的运用; 八、提高信息资源的利用; 九、 加强师生、生生互动与交流; 十、增强物理与医学的学科关联性。然而随机抽取乙班 12 名学生进行面谈, 他们对于传统教学的效果并不是特别满意。

在翻转课堂教学过程中, 教师未直接参与每个专题的讲授, 但教师要对每一个小组的教学设计和试讲 给予指导。通过这一过程可增进师生友谊, 加强师生交流, 还有利于提升学生的理论联系实际、分析解决 问题、归纳总结、批判性思维、创新思维、团队合作、沟通表达等能力, 使学生的综合素质得到拓展。

翻转课堂教学效果最直接的反映为甲乙两个班的考试成绩的对比。明显可见, 实验组的理论、实验考 试成绩均比对照组的成绩高, 对比差异满足统计学规律 $(P<0.001)$ 。见表 2 。

表 2 实验组与对照组期末成绩的对比

\begin{tabular}{lccc}
\hline 组名称 & 学生人数 & 理论考试成绩 $($ 分 $)$ & 实验考试成绩 $($ 分 $)$ \\
\hline 实验组 & 48 & $85.85 \pm 7.51$ & $96.35 \pm 6.35$ \\
对照组 & 51 & $80.12 \pm 5.43$ & $93.31 \pm 3.52$ \\
$\mathrm{t}$ & & 4.11 & 5.67 \\
$\mathrm{P}$ & & $<0.001$ & $<0.001$ \\
\hline
\end{tabular}

\section{4 总结}

翻转课堂促进了教师角色、教学环节、教学管理、学习方式、评价机制等的转变, 其在医学物理学教 学中的应用是一项全新的探索。翻转课堂在提高学生成绩、提升教学效果、拓展学生综合能力等方面都有 不可估量的作用。通过与传统课堂进行对比结果表明翻转课堂在医学物理学课程中实施具有一定的可行 性。为了不断提高翻转课堂的教学效果, 不断利用校内外各种有利资源和现代教育技术升级课堂教学是必 不可少的途径。

\section{6 致谢}

本文由湖北医药学院研究生科研启动金项目资助，项目编号：2014QDJZR17。

\section{Acknowledgement}

This work was supported by the initial project for postgraduates of Hubei University of Medicine (NO. 2014QDJZR17).

\section{参考文献:}

[1] 穆爱霞. 医学物理学开设问题探究 [J]. 大学物理实验, 2015,28 (5): 132-135.

[2] 李光仲, 刘俊英, 王云创等。基于医学物理学应用的《医用物理学》教学内容改革与实践[J]. 中国医学教育技术, 2016, 30 (1): 115-117.

[3] 褚巧燕, 袁小燕. 提高医学物理学教学质量的体会[J]. 包头医学院学报, 2011,27 (3): 115-117.

[4] 王亚平. 医学物理学教学中的案例教学法探析[J]. 中国医学物理学杂志, 2010,27 (1): 1696-1698.

[5] 武继文, 卢礼萍. 以临床应用为案例的医学物理学教学实践研究 [J]. 基础医学教育, 2014,16 (11): 915-917.

[6] 陈晓菲.翻转课堂教学模式的研究[D]. 武汉: 华中师范大学, 2014.

[7] 韦燕.“微课”视域下的翻转课堂教学模式创新研究[J]. x 信息与电脑, 2016, (1): 247-248. 
[8] 张鹏程, 吴静, 朱本超. 《医学物理学》医用物理部分课程内容的整合 [J]. 中国医学物理学杂志, 2009,26 (6): 1565-1566.

[9] 颜红金. 医学物理学与医学课程相结合的研究[J]. 中国医学物理学杂志, 2014,31 (1): 4718-4722.

[10] 杨淑改. 翻转课堂教学模式在预防医学教学中的应用效果评价[J]. 中国医学教育技术, 2016,30（5): 576-579.

\section{References}

[1] Mu Ai-xia: "Health development of medical physics"[J]. Physical Experiment of College, Vol. 28(2015), No.5, p.132-135 (in Chinese)

[2] Li Guang-zhong, Liu Jun-ying, Wang Yun-chuang, et al: “Teaching content reform and practice of Medical Physics based on medical application" [J]. Physical Experiment of College, Vol. 30(2016), No.1, p.115-117 (in Chinese)

[3] Chu Qiao-yan, Yan Xiao-yan: "To improve the quality of medical physics teaching”[J]. Journal of Botou medical college, Vol. 27(2011), No.3, p.115-117 (in Chinese)

[4] Wang Ya-ping: "The study on the case teaching of medical physics teaching" [J]. Chinese journal of medical physics, Vol. 27(2010), No.1, p.1696-1698 (in Chinese)

[5] Wu Ji-wen, Lu Li-ping: "Research on teaching practice of medical physics through cases of clinical application" [J]. Chinese journal of medical physics, Vol. 16(2014), No.11, p.915-917 (in Chinese)

[6] Chen Xiao-fei: "The study on flipped classroom teaching model" [D]. Wuhan: Central China Normal University, 2014. (in Chinese)

[7] Wei yan: "The study of flipped classroom teaching model innovation under micro-lecture perspective" [J]. China Computer Communication, Vol. 1(2016), p.247-248 (in Chinese)

[8] Zhang Peng-cheng, Wu Jing, Zhu Ben-chao: "Concordance of course content for medicine in medical physics" [J]. Chinese journal of medical physics, Vol. 26(2009), No.6, p.1565-1566 (in Chinese)

[9] Yan Hongjin: "Study on combination of medical physics and medical courses" [J]. Chinese journal of medical physics, Vol. 31(2014), No.1, p.4718-4722 (in Chinese)

[10] Yan Shugai: "Evaluation of the flipped classroom model applied in preventive medicine teaching" [J]. China education technology, Vol. 30(2016), No.5, p.576-579 (in Chinese) 